Investigation of reliability of the cutoff probe by a comparison with Thomson scattering in high density processing plasmas

Byonghoon Seo, Dae-Woong Kim, Jung-Hyung Kim, and Shinjae You

Citation: Physics of Plasmas 24, 123502 (2017);

View online: https://doi.org/10.1063/1.4996220

View Table of Contents: http://aip.scitation.org/toc/php/24/12

Published by the American Institute of Physics

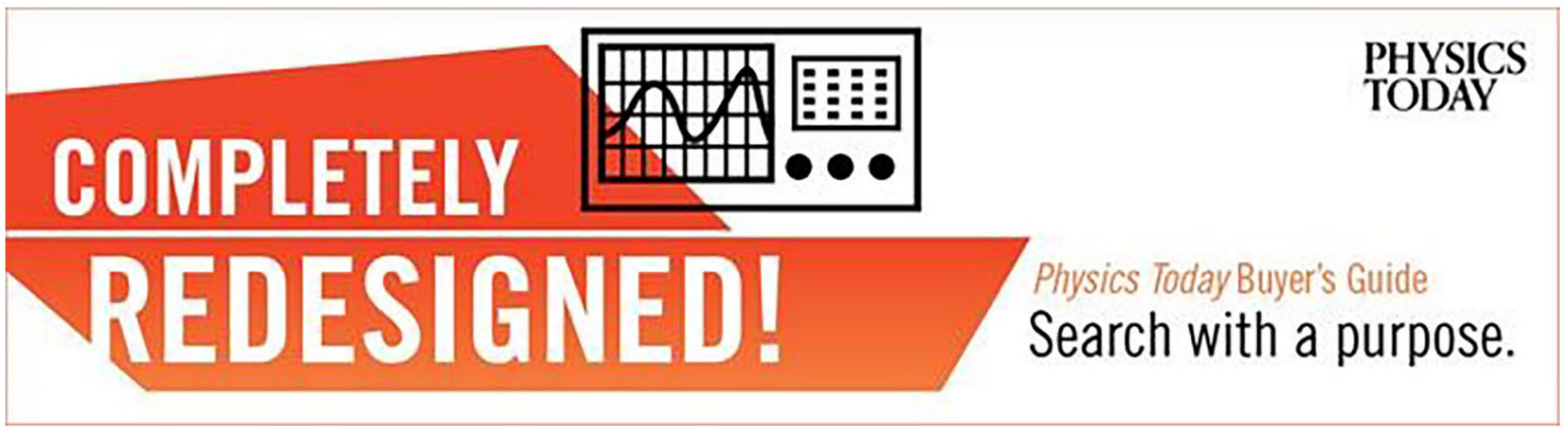




\title{
Investigation of reliability of the cutoff probe by a comparison with Thomson scattering in high density processing plasmas
}

\author{
Byonghoon Seo, ${ }^{1}$ Dae-Woong Kim, ${ }^{2}$ Jung-Hyung Kim, ${ }^{3}$ and Shinjae You ${ }^{4, a)}$ \\ ${ }^{1}$ Applied Physics and Materials Science, Caltech, Pasadena, California 91125, USA \\ ${ }^{2}$ Korea Institute of Machinery and Materials, Daejeon 34103, South Korea \\ ${ }^{3}$ Center for Vacuum Technology, Korea Research Institute of Standards and Science, Daejeon 34113 , \\ South Korea \\ ${ }^{4}$ Department of Physics, Chungnam National University, Daejeon 34134, South Korea
}

(Received 14 July 2017; accepted 8 November 2017; published online 6 December 2017)

\begin{abstract}
A "cutoff probe" uses microwaves to measure the electron density in a plasma. It is particularly attractive because it is easy to fabricate and use, its measurement is immune to surface contamination by dielectric materials, and it has a straightforward analysis to measure electron density in real time. In this work, we experimentally investigate the accuracy of the cutoff probe through a detailed comparison with Thomson scattering in a low temperature, high density processing plasma. The result shows that the electron density measured by the cutoff probe is lower than that by Thomson scattering and that the discrepancy of the two results becomes smaller as the gap between the two tips increases and/or the neutral gas pressure decreases. The underestimated electron density found by the cutoff probe can be explained by the influence of the probe holder, which becomes important as the pressure increases and the gap gets closer. Published by AIP Publishing. https://doi.org/10.1063/1.4996220
\end{abstract}

\section{INTRODUCTION}

The semiconductor industry has been growing rapidly with the next generation of flash memory. With these great technological advances, more precise processes have become necessary during etching and deposition. ${ }^{1}$ To support this, much research has been focused on improving precision processing technology. Relatedly, many diagnostic methods have been proposed and developed for study as a means to more accurately measure plasma parameters, such as electron density and temperature, which directly affect the results of the processing. Since accurate diagnosis of these plasma parameters is important, it has been continually investigated by comparative studies. $^{2-5}$

Diagnosis using probes has often been employed because the types and methods of these diagnostics are easy to make, use, and utilize in both the laboratory and industry. The most famous and often used is the Langmuir probe. ${ }^{6}$ It is one of the most powerful and well-developed tools for measuring plasma parameters such as plasma density, temperature, and plasma potential. There is also a criterion for using the probe in various circumstances of plasmas. ${ }^{6,7}$ Comparative studies have also been performed with other diagnostic methods such as Thomson scattering (TS) and optical emission spectroscopy, which means that the reliability of the probes has been investigated to a great extent. ${ }^{2-4}$

Over the past few decades, many probes that use microwaves have also been operated in conjunction with Langmuir probes. The plasma parameters can be measured even if the probe is contaminated by dielectric materials during the process, so it can be applied to processing plasmas. ${ }^{8-11}$ As mentioned in the abstract, we are interested in

\footnotetext{
${ }^{a)}$ Email: sjyou@cnu.ac.kr
}

the "cutoff probe (CP),"12 which utilizes the "cutoff" effect at which the electromagnetic wave is reflected, a quantity easily related to the electron density. The probe can measure the plasma frequency and thus plasma density in real time. It can also measure the electron-neutral collision frequency ${ }^{13}$ and the electron density in a pulsed plasma by the "Fourier cutoff" ${ }^{\prime 14}$ and can be used to study the plasma sheath. ${ }^{15,16}$ The CP has been theoretically studied and confirmed since it has been investigated through computer simulation and a simple circuit model. ${ }^{15,17}$ The main disadvantage of using this probe is that it interferes with the plasma because the probe is immersed inside it which inevitably affects the plasma. The accuracy of the cutoff probe has been numerically verified, but the simulation did not take into account all the effects of actual plasma conditions such as distortion of the sheath. Moreover, recently, the dispute related to the microwave probe arises from unrealistic and ambiguous assumptions in models. ${ }^{8}$

In this study, the reliability of $\mathrm{CP}$ is investigated by a comparison with TS under various conditions. TS is known to be the most reliable method and is used as a standard method in various comparative studies since the effect of the laser on plasmas is negligible. ${ }^{2-4,18}$

\section{EXPERIMENTAL SETUP AND METHODS}

Figure 1(a) shows a schematic of the experimental setup. A cylindrical stainless steel vacuum chamber with an inner diameter and a height of 300 and $220 \mathrm{~mm}$, respectively, is covered with a cylindrical ceramic plate with a diameter and a thickness of 280 and $15 \mathrm{~mm}$, respectively. The plasma is ignited and sustained by a single-turned antenna with 13.56 MHz RF current supplied from a power supply (up to $1000 \mathrm{~W}$ ) through an L-type impedance matching network 


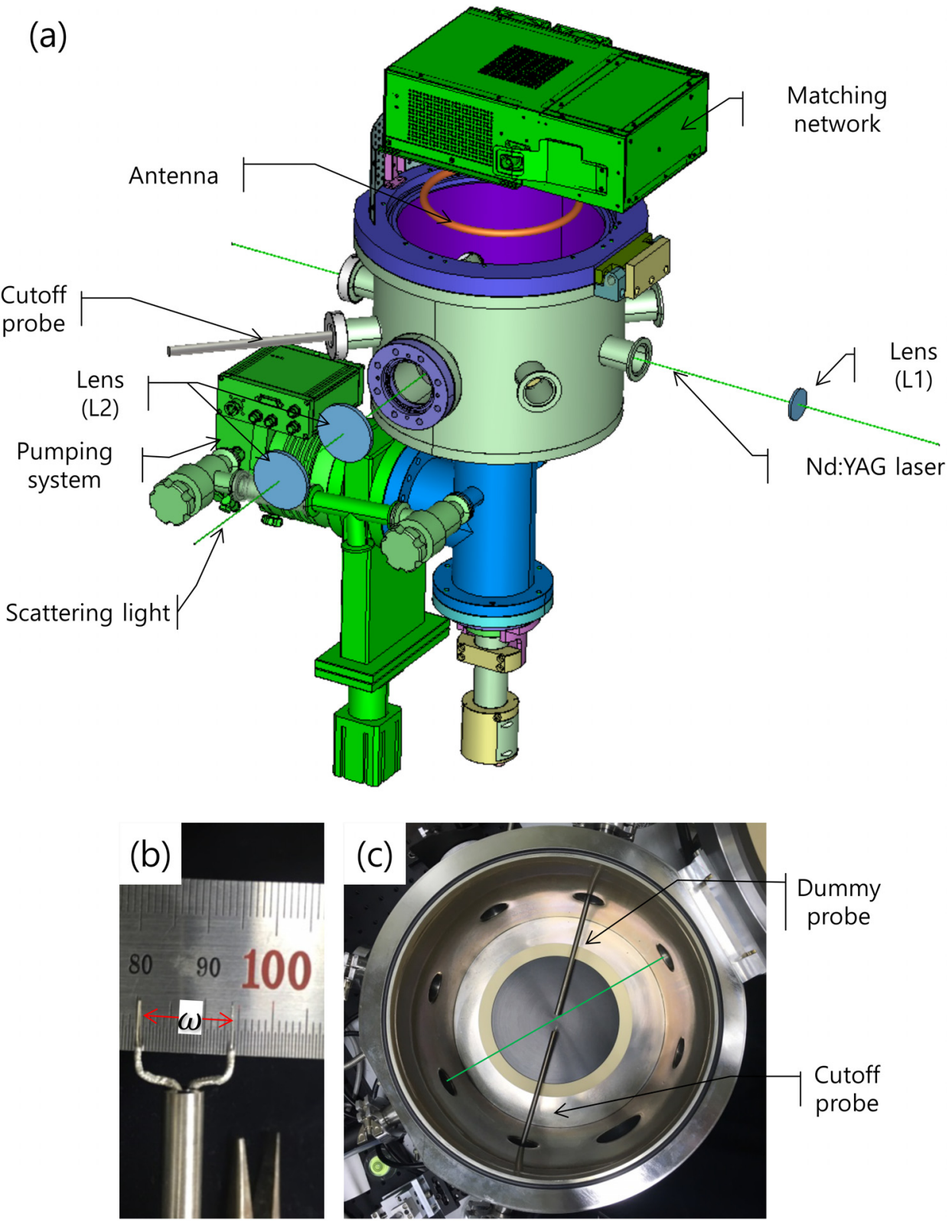

FIG. 1. Schematic of the experimental setup for TS and CP in ICP. and coaxial cable. Argon gas is supplied during discharge, and a capacitive manometer (MKS Inc.) was installed to monitor the gas pressure.

The CP consists of two thin coaxial cables inserted into a stainless-steel tube with an outer diameter of $1 / 4$ in. as shown in Fig. 1(b). The cable core at the end was exposed to the plasma at the measurement location. The CP measures the transmission magnitude spectrum (S21) using a network analyzer and collects signals between two tips immersed in the plasma (one for radiation and the other for detection). The spacing between the tips is determined as shown in Fig. 1(b). The experiment was carried out by varying the gap width. As shown in Fig. 1(c), the dummy probe was used with the $\mathrm{CP}$ when looking at the effect of the probe itself. For a more detailed description of the principle and method of the CP, see Ref. 12.

Figure 2 shows the transmission spectra of CP measurements at (a) a relatively low electron density with a $2 \mathrm{~mm}$ tip gap width of the tips and (b) a relatively high electron density with a $14 \mathrm{~mm}$ gap width. The shape of the transmission spectrum changes from the N-shape [Fig. 2(a)] to the step-like shape [Fig. 2(b)]. The absolute electron density measured by the CP method can be deduced from the following equation:

$$
n_{e}=\left(\frac{f_{p e}}{8980}\right)^{2}\left[\mathrm{~cm}^{-3}\right]
$$

where $f_{p e}$ is the electron plasma frequency (called "cutoff frequency"). The transmission spectra were measured and averaged right before and after the TS measurement and at each condition.

For the TS experiment, we used a frequency-doubled $\mathrm{Nd}$ : YAG laser operating at $10 \mathrm{~Hz}$ with a pulse energy of $350 \mathrm{~mJ}$ at $532 \mathrm{~nm}$. The vertically polarized laser beam was focused at the center of the discharge chamber where the experiment was performed using lens L1 (focal length $f=700 \mathrm{~mm}$ ). The TS signal was collected by a pair of 

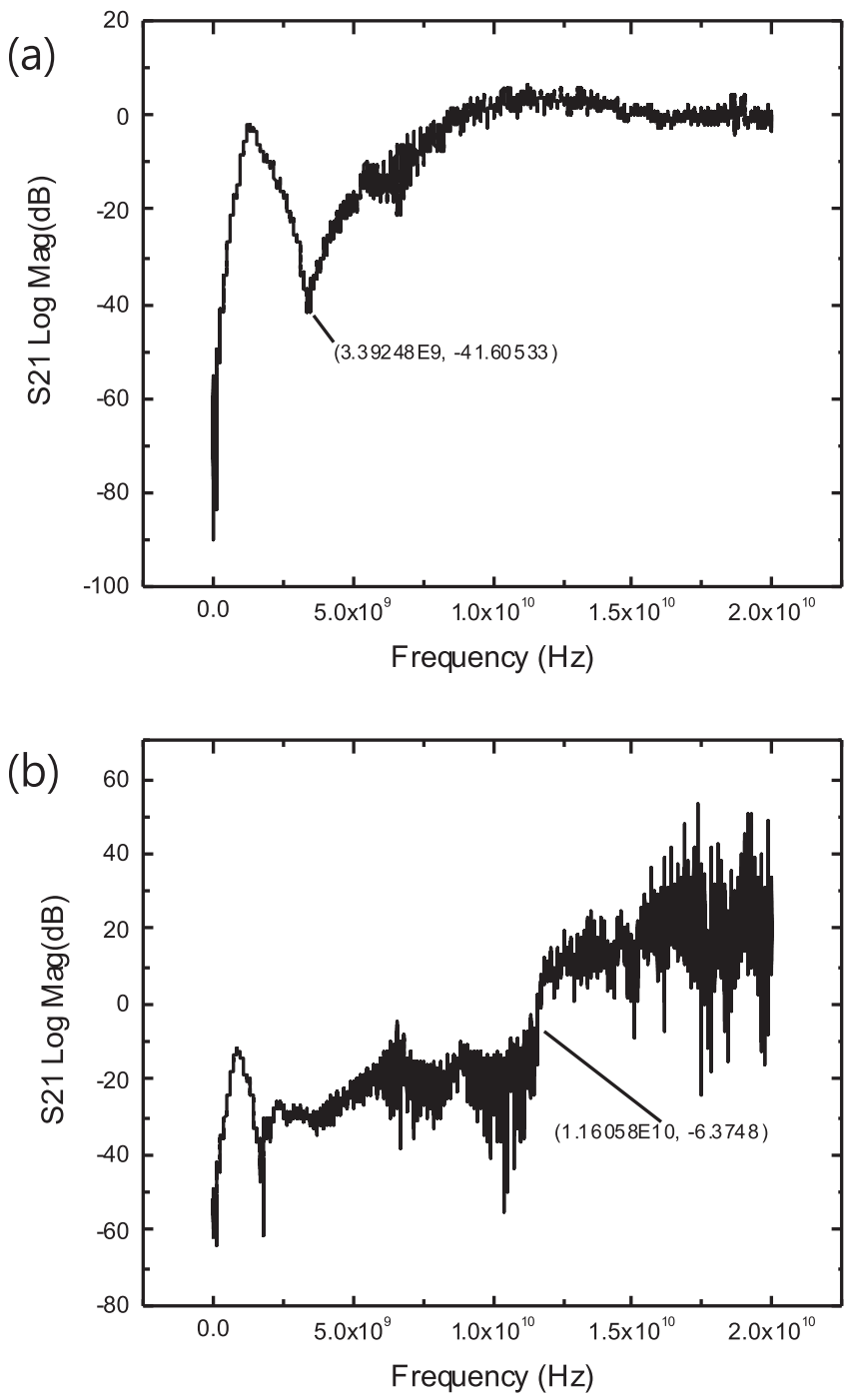

FIG. 2. Measured CP spectrum at (a) low electron density with a $2 \mathrm{~mm}$ gap width and (b) high electron density with a $14 \mathrm{~mm}$ gap width of the tips.

achromatic lenses (L2) at $90^{\circ}$ from the laser direction in which the scattering signal is maximized. One of the achromatic lenses was used to collimate scattered light, and the other was to focus on the entrance slit $(250 \mu \mathrm{m})$ of the double monochrometer. In order to block the Rayleigh scattering (RS) and stray light (ST) signals, a Rayleigh mask with a thin carbon lead of $0.3 \mathrm{~mm}$ was installed in the double monochrometer (two monochrometers are used in series, and the mask is installed between an exit of the first monochrometer and an entrance of the second monochrometer). Using an ICCD camera, we amplified and accumulated the signals. The ICCD camera was operated at a doubled trigger signal $(20 \mathrm{~Hz})$, twice the laser trigger $(10 \mathrm{~Hz})$, which subtracts the background signal emitted from the plasma simultaneously. Strictly speaking, the time difference between the TS signal and the background plasma signal is $50 \mathrm{~ms}$, and since continuous RF power is used, it is assumed that the plasma emission for $50 \mathrm{~ms}$ is constant over time.

The focal length of the spectrometer and lenses is $300 \mathrm{~mm}$ to match the numerical aperture and hence one to one magnification. The size of a CCD pixel is $13 \mu \mathrm{m}$ $\times 13 \mu \mathrm{m}, 512$ pixels were used with binning vertically, and so, the spatial resolution is about $6 \mathrm{~mm}$.

Laser energy was monitored at the end of the laser path using a power meter during the measurement time to compensate for the temporal variation of the signal induced from different laser intensities. Details about the Thomson scattering setup and the estimation of the uncertainty can also be found in Refs. 4 and 18.

In our experiment, the TS is in a non-collective regime because of the scattering parameter, $\alpha \sim \lambda / \lambda_{D e} \ll 1$, where $\lambda$ is a wavelength of the laser and $\lambda_{D e}$ is the Debye length. ${ }^{19}$ Figure 3 shows a TS spectrum measured at 100 mTorr and $950 \mathrm{~W}$, and a ST spectrum measured at the base pressure can be seen. Because the mask was installed in the monochrometer, the ST and RS signals were almost blocked at the center of the TS spectrum. Figure 4(a) shows the measured ST and RS spectra, and Fig. 4(b) shows the integrated signal intensity of the spectrum of Fig. 4(a). The RS signal was measured as a function of pressure without a mask installed. The signal varies linearly with pressure and is used to obtain an absolute electron density. The pure TS and RS signals were obtained by subtracting the ST signal with and without installing the mask in the monochrometer, respectively. The laser scattering signal was measured and recorded by accumulation of over 2000 laser shots taken for about $200 \mathrm{~s}$ and repeated three times except for $20 \mathrm{mTorr}$. At 20 mTorr, the laser scattering signal was accumulated over 4000 laser shots taken for about $400 \mathrm{~s}$ and repeated 3 times due to the low signal-to-noise ratio. The absolute electron density measured by the TS can be obtained by the following equation:

$$
n_{e}=n_{g} \frac{P_{P T S}}{P_{P R S}} \Gamma_{R, A R},
$$

where $P_{P T S}$ is the integrated pure TS signal, $P_{P R S}$ is the integrated pure RS signal, and $\Gamma_{R, A r}=6.80 \times 10^{-3}$ for argon. ${ }^{20}$ $n_{g}$ is the neutral density expressed by the ideal gas law as $n_{g}=p /\left(k_{B} T_{g}\right)$, where $k_{B}$ is the Boltzmann constant, $T_{g}$ is the neutral gas temperature set to a room temperature, and $p$ is

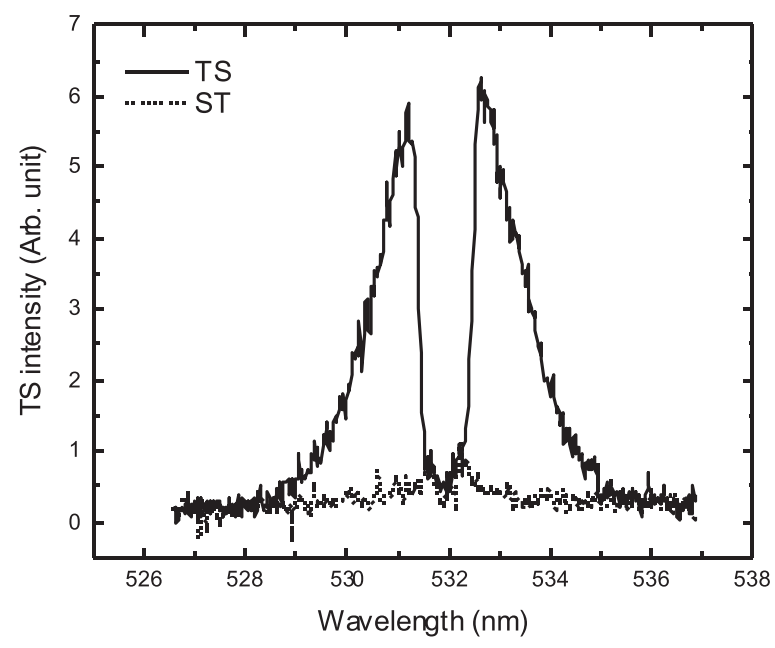

FIG. 3. Measured pure TS and ST spectra. 
(a)

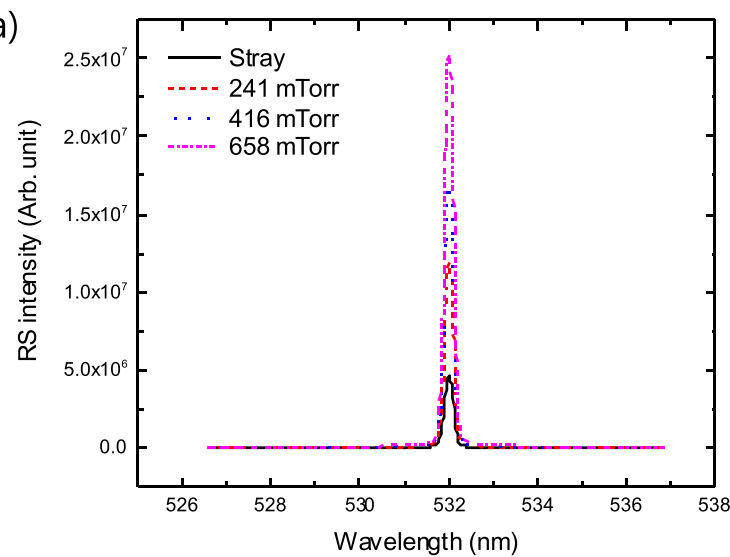

(b)

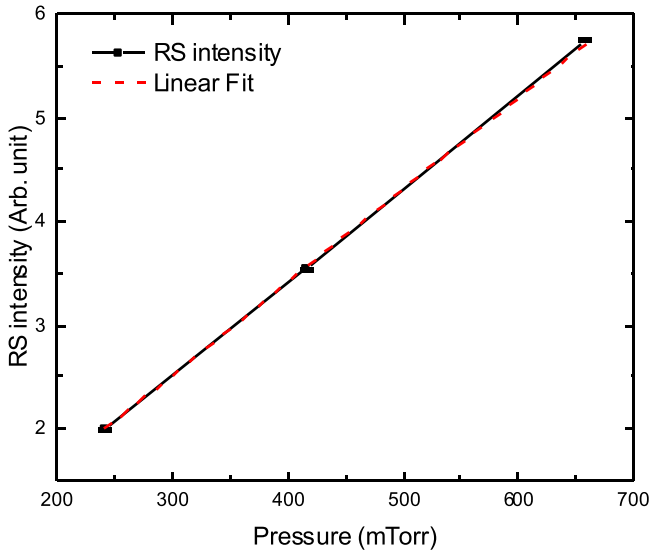

FIG. 4. (a) Measured RS and ST spectra in pressure and (b) integrated signals in pressure.

the gas pressure at which the measurement of the RS signal is done.

\section{RESULTS AND DISCUSSION}

Figure 5 shows the electron densities measured by the $\mathrm{CP}$ plotted in the horizontal direction and by the TS in the vertical according to different gas pressures with a $14 \mathrm{~mm}$ gap width between the tips. The line indicated as " $Y=X$ " means that the electron densities measured by both methods

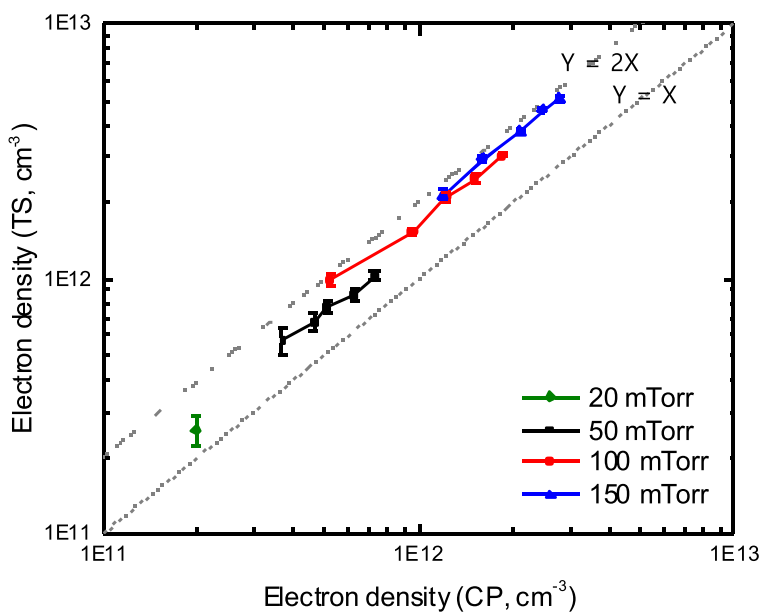

FIG. 5. Measured electron densities using the TS and CP as a function of gas pressure. are identical and " $Y=2 X$ " means that the electron densities measured by the TS are twice the CP. At 150 mTorr, the electron densities measured by the TS are approximately 1.8 times greater than these measured by the $\mathrm{CP}$. As the gas pressure goes down to $20 \mathrm{mTorr}$, the electron densities measured by the CP become closer to those measured by the TS. At 20 mTorr, the electron densities measured by the TS are approximately 1.3 times greater than these measured by the CP. Unfortunately, not enough data were obtained at 20 mTorr because the electron density was saturated even if more rf power was applied and the lowest detection limit for the TS in our system was reached. It is clear that the discrepancy between the results is caused by the gas pressure.

Figure 6 shows the experimental results of the TS and CP with different gap widths of the CP tip at 100 mTorr Ar. The results show that the electron density measured by the CP gets closer to the TS as the gap becomes wider. This means that as the gap narrows, the CP measures the density to be lower.

The results shown in Figs. 5 and 6 can be explained as follows: In a $\mathrm{CP}$ measurement, the radiating microwave travels from one tip to the other. This means that the CP measures the plasma between the tips as depicted in Fig. 7. If the probe is immersed in the plasma, the density in the vicinity of the probe is depleted due to a loss by the probe surface, so the spatial distribution of the electron density can be formed as parabolic-like as depicted in Fig. 8. The cutoff probe measures in the depleted region. For the parabolic-like distribution of the density, a center to edge ratio, $n(R) / n(0)$, where $n(R)$ is the density at the edge and $n(0)$ is the density at the center, is proportional to square root of the ion mean free path, and the ion mean free path is inversely proportional to the pressure. ${ }^{1}$ If we assume that the density at the center is the same, the edge density is inversely proportional to the square root of the pressure. As the pressure increases, the ion mean free path gets shorter, and the edge density decreases. Therefore, the cutoff probe measures a lower density as the pressure increases.

If the tip width, $w$, becomes wider than the diameter of the probe, less-depleted plasma regions are included between

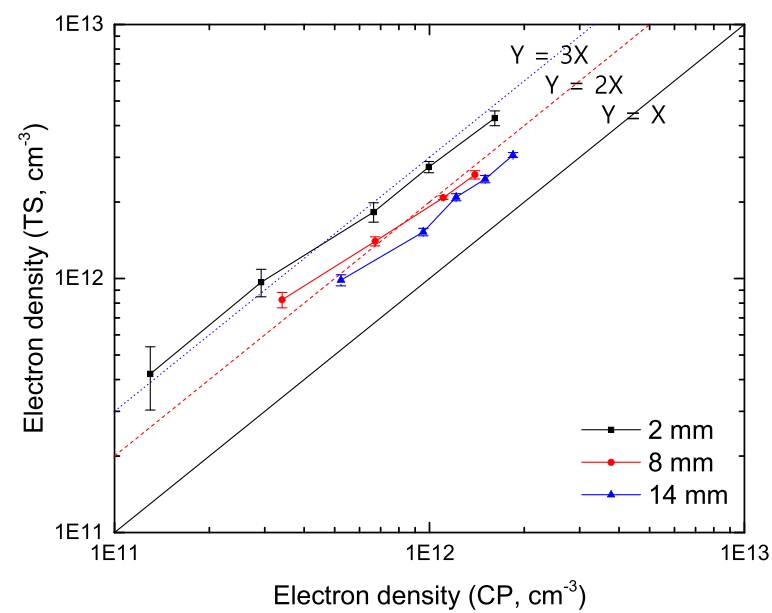

FIG. 6. Measured electron densities using the TS and $\mathrm{CP}$ as a function of the gap width of the tips. 


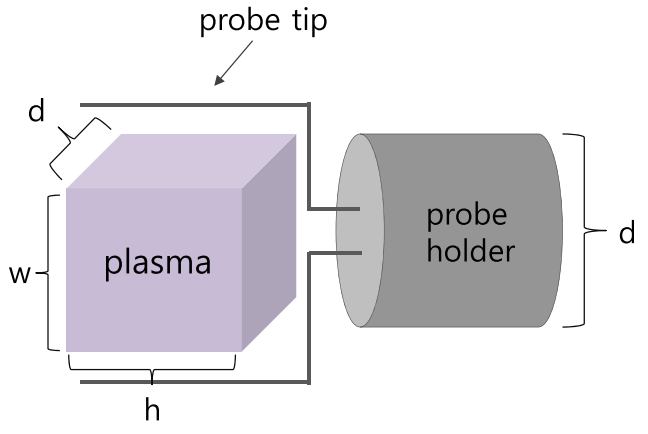

FIG. 7. Schematic of the depletion area in the vicinity of the probe.

the tips. Thus, the density measured by $\mathrm{CP}$ gets closer to the density measured by TS. Since the plasma loss in the vicinity of the probe is dominantly by the surface loss and the surface loss is proportional to the surface area, the ratio of the probe surface area to the surface area of the plasma between the tips increases as the plasma volume between the tips decreases. To confirm this, we calculate the ratio of the plasma surface area relative to the probe area based on Fig. 7. For simplicity, we assume that the plasma is rectangular and the length of the sheath is neglected, which will be discussed later. The plasma surface $S_{p}=2(h w+d(h+w))$, where $h$ is the height, $d$ is the depth, and $w$ is the width. We also assume that $d$ is the same as the diameter of the probe holder. The surface area of the probe holder $S_{h}=\pi(d / 2)^{2} . h$ is $10 \mathrm{~mm}, d$ is $6.35 \mathrm{~mm}(1 / 4 \mathrm{in}$.), and $w$ s are 2,8 , and $14 \mathrm{~mm}$. The ratios, $S_{h} / S_{p}$, are listed in Table I.

As shown in Table I, the smaller the gap, the larger the ratio. The ratio in the case where $w$ is $2 \mathrm{~mm}$ is about three times as large as that in the case of $14 \mathrm{~mm}$, and the ratio when $w$ is $8 \mathrm{~mm}$ is about 1.5 times as large as $14 \mathrm{~mm}$. The results are qualitatively in good agreement with the experimental results. As the gap shrinks, the ratio of the plasma surface to the probe holder surface decreases and the electron density is measured to a lower value.

Figure 9 shows the measured electron densities by (a) the TS and (b) by the $\mathrm{CP}$ when immersing a dummy probe at $20 \mathrm{~mm}$ from the measurement point of the Thomson scattering to see the effect of the probe holder. The pressure is set to $100 \mathrm{mTorr}$, and the tip width is $14 \mathrm{~mm}$. The results show that the electron density is lower when the dummy probe is immersed for both the CP and TS cases. It means that by immersing a probe, the probe itself makes plasmas have

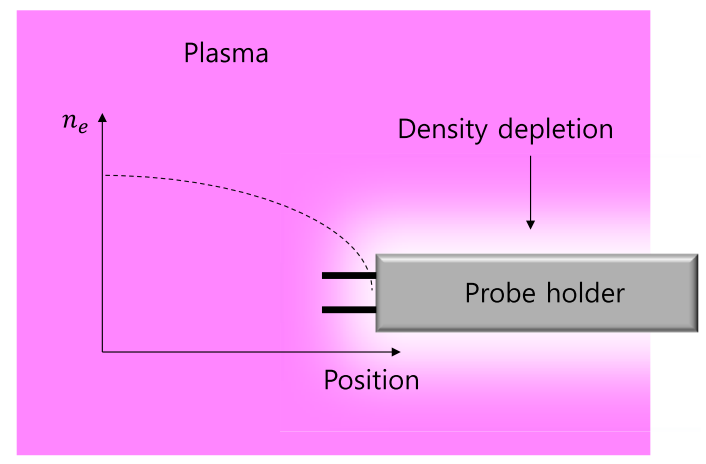

FIG. 8. Schematic of the immersed probe in the plasmas.
TABLE I. Surface area ratio.

\begin{tabular}{lc}
\hline \hline$w(\mathrm{~mm})$ & $S_{\mathrm{h}} / S_{\mathrm{p}}$ \\
\hline 2 & 0.1646 \\
8 & 0.0815 \\
14 & 0.0542 \\
\hline \hline
\end{tabular}

lesser density in the vicinity of the probe as depicted in Fig. 8. Since the stray light becomes more significant as the distance between the dummy probe and the laser gets closer, $20 \mathrm{~mm}$ is the minimum length for inserting the dummy probe, but if the distance between the dummy probe and the measurements gets shorter, the density would be measured to be lower.

Although we neglected the sheath length on the estimation of the surface ratio above, there might be an effect of the sheath when the gap width is comparable to the sheath length as documented by Godyak in Ref. 8. Although the sheath length cannot be accurately defined because there is no strict boundary between the plasma and the sheath, the sheath length can be estimated based on various models as $L_{s h}=n \lambda_{D e}$, where $\mathrm{n}$ is some constant value depending on the sheath model and $\lambda_{D e}$ is the Debye length defined as $\lambda_{D e}$ $=740 \sqrt{T_{e} / n_{e}}$ in $\mathrm{cm}$, where $T_{e}$ is the electron temperature in $\mathrm{eV}$ and $n_{e}$ is the electron density in $\mathrm{cm}^{-3} .{ }^{1}$ In our experimental

(a)

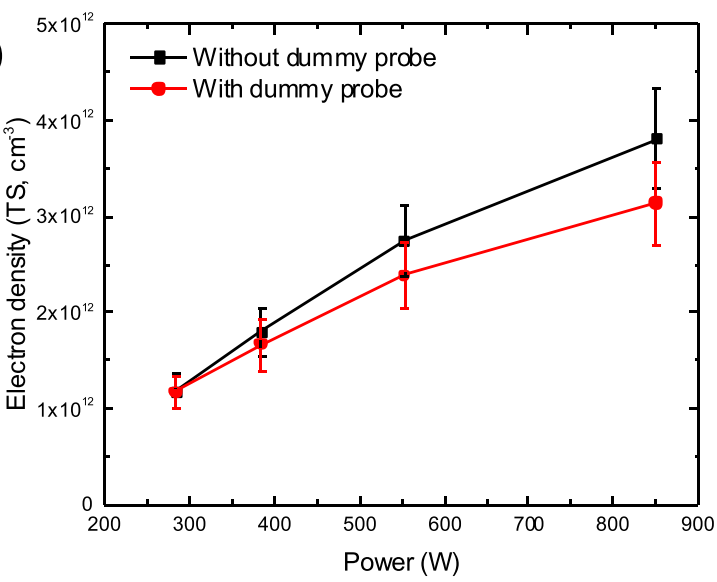

(b)

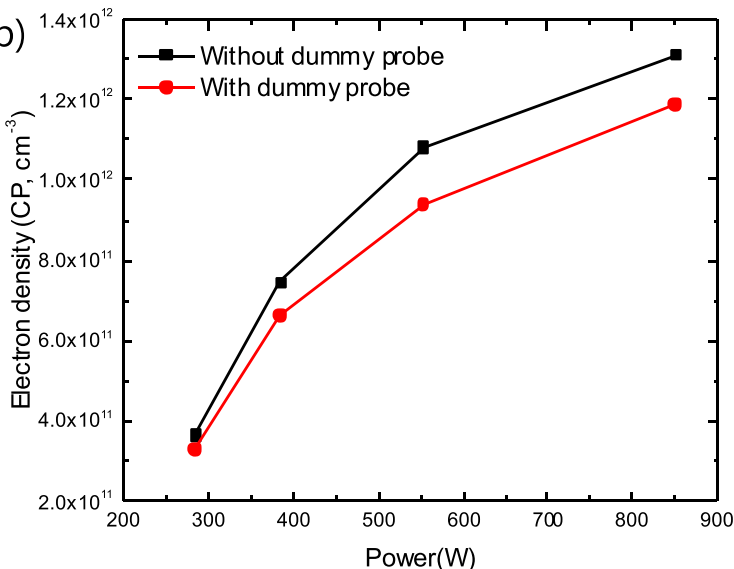

FIG. 9. Measured electron densities by the (a) TS and (b) CP with and without inserting a dummy probe, respectively. 
range where $T_{e}$ is around $2 \mathrm{eV}$ and $n_{e}$ is in the range of $1 \times 10^{11} \sim 1 \times 10^{13} \mathrm{~cm}^{-3}$, the Debye length is in the range of $3.3 \times 10^{-3} \sim 3.3 \times 10^{-2} \mathrm{~mm}$, which is much less than the width of the gap. We believe that the effect of the sheath length on this experiment is not significant for the $2 \mathrm{~mm}$ gap width and negligible for the other lengths, but it could be considerable when the electron density is low like in capacitively coupled plasmas where the sheath length is comparable to the gap width.

\section{CONCLUSIONS}

We have investigated the accuracy of the cutoff probe by a comparison with Thomson scattering in low temperature plasmas. The results show that the electron densities measured by the cutoff probe have lower values than those by the Thomson scattering and the discrepancy of the results becomes smaller as the pressure goes down and/or the gap between two tips becomes wider. The discrepancy between the methods can be explained by the influence of the probe holder that becomes significant as the pressure rises and the gap gets closer.

\section{ACKNOWLEDGMENTS}

This research was supported by the Korea Research Institute of Standards and Science, by the MOTIE [Ministry of Trade, Industry \& Energy (10052890 Numerical simulation to overcome process limitations below $10 \mathrm{~nm}$ semiconductor and 10053098 Plasma enhanced atomic-layer-deposition process and alternatives for gate spacer and multi-patterning technology)] by the KSRC (Korea Semiconductor Research Consortium) support program for the development of the future semiconductor device, by the Ministry of Science, ICT and Future Planning (NRF-2017M1A7A1A02016321 and NRF-2017R1D1A1A02018310), by the Program of the "2017 plasma BigData ICT Convergence Technology Research Project" through the National Fusion Research Institute of
Korea, by the Korea Research Institute of Standards and Science (Kriss-2017-GP2017-0016-01), and by the National Research Council of Science \& Technology (NST) grant by the Korea government (MSIP) (No. CAP-17-02NFRI).

${ }^{1}$ M. A. Lieberman and A. J. Lichtenberg, Principles of Plasma Discharges and Materials Processing (John Wiley \& Sons, 2005).

${ }^{2}$ M. Noguchi, T. Hirao, M. Shindo, K. Sakurauchi, Y. Yamagata, K. Uchino, Y. Kawai, and K. Muraoka, Plasma Sources Sci. Technol. 12, 403 (2003).

${ }^{3}$ D. L. Crintea, U. Czarnetzki, S. Iordanova, I. Koleva, and D. Luggenhölscher, J. Phys. D: Appl. Phys. 42, 45208 (2009).

${ }^{4}$ B. H. Seo, S. J. You, G. H. You, J. H. Kim, Y. S. Yoo, D. J. Seong, and H. Y. Chang, J. Instrum. 7, C01049 (2012).

${ }^{5}$ D. W. Kim, S. J. You, J. H. Kim, H. Y. Chang, and W. Y. Oh, Plasma Sources Sci. Technol. 25, 35026 (2016).

${ }^{6}$ V. A. Godyak, R. B. Piejak, and B. M. Alexandrovich, Plasma Sources Sci. Technol. 1, 36 (1992).

${ }^{7}$ V. A. Godyak, Plasma Phys. Controlled Fusion 45, A399 (2003).

${ }^{8}$ V. Godyak, Phys. Plasmas 24, 060702 (2017).

${ }^{9}$ K. Takayama, H. Ikegami, and S. Miyazaki, Phys. Rev. Lett. 5, 238 (1960).

${ }^{10}$ R. L. Stenzel, Rev. Sci. Instrum. 47, 603 (1976).

${ }^{11}$ M. Lapke, T. Mussenbrock, and R. P. Brinkmann, Appl. Phys. Lett. 93, 051502 (2008)

${ }^{12}$ J. H. Kim, D. J. Seong, J. Y. Lim, and K. H. Chung, Appl. Phys. Lett. 83, 4725 (2003).

${ }^{13}$ K. H. You, S. J. You, D. W. Kim, B. K. Na, B. H. Seo, J. H. Kim, and H. Y. Chang, Phys. Plasmas 23, 033509 (2016).

${ }^{14}$ B. K. Na, K. H. You, D. W. Kim, B. H. Seo, H. Y. Chang, S. J. You, and Y. S. Lee, J. Instrum. 7, C04022 (2012).

${ }^{15}$ D. W. Kim, S. J. You, J. H. Kim, H. Y. Chang, and W. Y. Oh, Plasma Sources Sci. and Tech. 25(3), 035026 (2016)

${ }^{16}$ D. W. Kim, S. J. You, J. H. Kim, H. Y. Chang, J. S. Yoon, and W. Y. Oh, Phys. Plasmas 23, 053516 (2016).

${ }^{17}$ D. W. Kim, S. J. You, B. K. Na, J. H. Kim, and H. Y. Chang, Appl. Phys. Lett. 99, 131502 (2011).

${ }^{18}$ B. H. Seo, S. J. You, and J. H. Kim, Jpn. J. Appl. Phys. 54, 086102 (2015).

${ }^{19}$ J. Sheffield, D. Froula, S. H. Glenzer, and N. C. Luhmann, Jr., Plasma Scattering of Electromagnetic Radiation: Theory and Measurement Techniques (Academic Press, 2010).

${ }^{20}$ A. W. DeSilva and G. C. Goldenbaum, Methods of Experimental Physics, Vol. 9, Part A (Academic, New York, 1970), p. 3. 